\title{
Multilinguales
}

\section{Le conte algérien des deux langues arabe et amazighe comme agent identitaire}

The algerian tale of the two languages arabic and amazighe, as an identity agent

\section{Attef Bouzidi}

\section{OpenEdition}

\section{Journals}

\section{Édition électronique}

URL : https://journals.openedition.org/multilinguales/4467

DOI : $10.4000 /$ multilinguales. 4467

ISSN : 2335-1853

Éditeur

Université Abderrahmane Mira - Bejaia

Référence électronique

Attef Bouzidi, « Le conte algérien des deux langues arabe et amazighe comme agent identitaire », Multilinguales [En ligne], 12 | 2020, mis en ligne le 14 février 2020, consulté le 30 juin 2021. URL : http:// journals.openedition.org/multilinguales/4467; DOI : https://doi.org/10.4000/multilinguales.4467

Ce document a été généré automatiquement le 30 juin 2021.

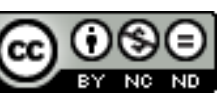

Multilinguales est mise à disposition selon les termes de la Licence Creative Commons Attribution Pas d'Utilisation Commerciale - Pas de Modification 4.0 International 


\title{
Le conte algérien des deux langues arabe et amazighe comme agent identitaire
}

The algerian tale of the two languages arabic and amazighe, as an identity agent

\author{
Attef Bouzidi
}

1 Cette citation décrit une réalité dont les enjeux peuvent aller bien au-delà d'une simple conception théorique du fait éducatif. Cette "pierre angulaire» devrait, dans le meilleur des mondes, pouvoir participer, d'abord, à la construction d'un individu indépendant dans ses convictions, responsable dans ses jugements et dans ses prises de décisions et surtout, conscient de cette indépendance et du poids de cette responsabilité.

Une «projection dans le futur » qui devrait être le moteur de toute société qui aspire à la continuité. Une continuité que devrait protéger un système identitaire qui trouve lui-même les fondements de ses motivations et de ses agissements présents dans une connaissance solide et consciente du " passé ».

Cette construction de l'« avenir » devrait se faire dans la prise en compte de tout ce qui peut animer la société comme "tendances" et orientations à la fois économiques, politiques, idéologiques, linguistiques et certainement sociales. Car il s'agit, en réalité, d'un avenir qui devrait permettre à la société de se positionner par rapport à sa propre histoire et à celle du monde.

2 Tout système éducatif devrait se concevoir, dans et par rapport, à l'intelligence et à la mémoire de la société, et notamment dans toutes leurs manifestations. Autrement dit, il ne pourrait y avoir de système éducatif performant et bénéfique pour la société que lorsqu'il serait conçu, d'un côté, dans le respect de ses spécificités sociales, culturelles et civilisationnelles, et d'un autre côté, dans l'objectif de lui donner la possibilité et la capacité de construire son avenir et notamment d'en avoir le contrôle.

3 Le conte en tant que l'un des produits de l'intelligence sociale et l'un des outils de sauvegarde de la mémoire collective pourrait faire partie des outils pédagogiques de 
l'éducation. Issu de l'oral, comme le confirme toutes les traditions savantes et universitaires, le conte a fait son introduction à l'écrit et notamment à l'écrit littéraire des siècles auparavant (Charles Perrault, Les Frères Grimm). Son exploitation dans l'univers éducatif, et notamment dans l'apprentissage des langues, a également fait l'objet de beaucoup d'exercices et d'expériences. Il se trouve désormais dans les divers paliers des systèmes éducatifs, servant comme support pédagogique dans la transmission, non seulement des savoirs et des connaissances, mais également des valeurs et des principes de vie.

Le caractère universel du conte a fait de lui l'un des outils pédagogiques les plus aptes à voyager à travers les cultures et les civilisations. Il a pu se proposer, par son essence même, comme un support éducatif très efficace et plein de richesses et de possibilités. Une essence qui a fait que le système éducatif algérien a pris soin d'introduire le conte comme support pour les diverses leçons et pour les divers exercices et ce dès le cycle primaire. Ce choix et cette orientation seraient animés par des aspirations bien pratiques. Permettre, d'un côté, l'apprentissage d'une langue étrangère et la familiarisation avec ses potentialités, et d'un autre côté, faire cet apprentissage à travers un genre littéraire qui peut toucher aux grandes réalités naturelle et humaine avec des mots simples et dans des univers que l'enfant préfère et adore.

Aussi, « le conte n'est pas simplement une mise en scène de l'histoire des hommes; c'est un jeu cosmique qui reprend les grands mythes de la nature... Le conte a donc pu être une manière voilée de parler des choses sacrées, une manière de mettre les grandes vérités à la portée de tous » (N'SOUGAN AGBLEMAGNON , 1986 : 18-19).

Il s'agit d'un produit social et culturel qui reprend, sur un registre à la fois naïf mais remarquablement responsable et moralisateur, les diverses réalités qui font le monde. Et c'est, par ailleurs, pour l'enfant, l'occasion de rencontrer des thèmes qui peuplent son imaginaire et qui sont également les sources de ses rêves et de ses préoccupations.

La prise en compte, dans l'acte pédagogique, de la dimension culturelle et civilisationnelle du conte pourrait être un moyen qui aiderait à « former les apprenants à l'esprit de la citoyenneté (l'histoire du pays, les valeurs sociales et culturelles, etc.) 》 (KHEIR et al., 2013: 54). Cet esprit de la citoyenneté devrait avoir pour valeur principale la participation et la contribution à la construction d'un monde et d'un mode de vie cohérent et ouvert. Cohérent dans ses principes et dans ses convictions, et ouvert dans sa réception des autres cultures et des autres modes de vie sans être ni dans l'imposition ni dans la permissivité sans réflexion.

Vu sous cet angle, le conte en tant que genre littéraire exploité dans l'acte pédagogique, acquiert une importance cruciale et une dimension, d'abord psychologique, puis référentielle et certainement identitaire. Nous essayerons dans cet article de nous arrêter sur l'apport que peut avoir l'introduction du conte dans l'acte pédagogique et notamment sur la constitution de la dimension identitaire chez les élèves du cycle primaire. Loin de nous concentrer ici essentiellement sur les côtés didactique et pédagogique de la mise en place d'un enseignement qui exploiterait le conte dans l'apprentissage des langues, nous nous proposerons dans cet article de revenir sur le rôle identitaire que peut jouer la présence du conte, et plus particulièrement du conte algérien (des deux langues: arabe et amazighe ${ }^{1}$ ), dans les programmes du cycle primaire de l'école algérienne.

5 Le présent article se propose comme ambition de répondre à une interrogation bien définie. L'Algérie reconnait désormais l'officialisation de deux langues nationales: l'arabe et l'amazighe (Constitution, 2016). Quel pourrait être l'apport identitaire de 
l'exploitation du conte algérien des deux langues arabe et amazighe dans l'enseignement du français (comme langue étrangère) au Cycle primaire?

Ce qui anime notre réflexion, c'est essentiellement le poids et l'impact que pourrait avoir l'exploitation du conte algérien (des deux langues: arabe et amazighe) dans le cycle primaire, et ce dans le processus psychologique et identitaire qui prépare le citoyen algérien de demain. Notre article se trouve motivé notamment par des changements réellement sérieux qui touchent de manière très profonde la société algérienne dans ses organisations culturelle et civilisationnelle. Une société algérienne qui ne peut être pensée loin d'un environnement mondial gouverné, depuis quelques années maintenant, par des conflits qui sont pratiquement sur tous les plans: économique, idéologique, politique et même militaire. Un environnement mondial animé et motivé, plus que jamais, par des questionnements identitaires que connaissent les différents pays.

6 La mondialisation a rendu les questions de l'appartenance et de l'identité des sujets incontournables lors de toutes les manifestations politique, philosophique, éducative et notamment idéologique dans presque tous les pays. Autant ces questions sont légitimes, autant sont légitimes toutes les réflexions qui se proposent de contrer des incompréhensions et des confusions qui peuvent aboutir, dans le pire des cas, sur des conflits physiques.

Le recours à l'exploitation du conte algérien des deux langues : arabe et amazighe à l'école primaire (un parcours qui s'étale pratiquement de l'âge de six ans jusqu'à l'âge de onze ans et où l'enseignement de la langue française commence en troisième année, à l'âge de huit ans) pourrait être une démarche très importante pour l'élève algérien. C'est une démarche qui lui permettrait, d'un côté, d'apprendre et d'affronter une langue étrangère et, d'un autre côté, de commencer, à travers cet apprentissage, par une prise de conscience de sa propre société de son appartenance et de sa propre culture. Il lui serait alors plus approprié et plus conscient de recevoir, au cycle suivant (au cycle moyen), par le biais des contes d'origines étrangères que peuvent comporter les manuels et les programmes éducatifs, d'autres contenus sociaux et culturels.

7 Loin de nous l'idée de vouloir renfermer les élèves algériens dans un espace culturel clos qui n'accepte pas l'altérité, ou encore de les isoler des autres références culturelles qui peuvent exister à travers la présence des contes étrangers. Mais ce qui pourrait être proposé par le recours aux contes algériens, ce serait une mise en place d'une conception assez forte et assez profonde des spécificités de la société algérienne pour pouvoir constituer une immunité contre toute volonté d'influence et de manipulation. L'ouverture, alors, sur d'autres sociétés, sur d'autres modèles culturels et civilisationnels se ferait à travers une conscience solide et bien enracinée.

Les programmes du cycle primaire de l'école algérienne contiennent actuellement des contes qui appartiennent à différentes origines et à diverses sphères culturelles. Ils ont leurs places servant comme support pédagogique pour l'apprentissage du français, première langue étrangère en Algérie,

«un statut qui a été, par ailleurs, à un certain moment donné de l'histoire de l'Algérie, remis en cause en faveur d'une arabisation qui voulait s'imposer comme élément identitaire unique, exclusif et incontournable pour l'individu algérien » (GRANDGUILLAUME, 2001 : 273-287).

Ces programmes proposent plusieurs contes d'appartenances et de cultures diverses. Ces contes sont exploités, d'abord, comme outil pédagogique dans l'enseignement 
d'une langue étrangère, puis dans l'objectif d'introduire et d'initier l'enfant aux structures formelle et narrative du conte en tant que genre littéraire.

8 Le système éducatif algérien a pris soin de les introduire à travers les diverses réformes qui ont marqué le paysage éducatif algérien depuis l'Indépendance (1962) jusqu'à nos jours. Mais il nous semble qu'une démarche qui s'appuierait d'abord sur la présence des contes algériens (des deux langues arabe et amazighe) et qui prendrait ces contes comme point d'ancrage identitaire, permettrait à l'élève de trouver facilement son chemin vers l'apprentissage de la langue française. Elle lui permettrait aussi, et c'est ce qui motive notre réflexion dans le présent article, de reconnaître dans ces contes son appartenance et de façonner, dès son jeune âge, une référence identitaire bien claire et bien précise.

Afin de pouvoir répondre à notre problématique, nous nous sommes proposé de vérifier la présence des contes algériens des deux langues (arabe et amazighe) dans les manuels du cycle primaire des années : troisième (2013-2014/2017-2018), quatrième et cinquième (2013-2014) de l'enseignement de la langue française.

Une lecture de notre corpus constitué des manuels algériens de l'école primaire, ceux qui concernent en l'occurrence l'apprentissage de la langue française, nous a permis de nous rendre compte d'une réalité étonnante. Il est, effectivement, surprenant que dans une Algérie aussi riche en productions orales et en traditions populaires, les programmes scolaires ne soient pas conçus et pensés dans une démarche qui exploiterait et qui mettrait en avant un patrimoine oral extrêmement présent dans la vie de tous les jours. Il nous a été possible de constater que le manuel de troisième année $e^{2}$ par exemple, ne propose aucun conte algérien. Le manuel de lecture de quatrième année ne se réfère, lui aussi, à aucun conte. Tandis que le livre des activités, contient deux contes avec l'indication « conte algérien $»^{3}$.

Le manuel de cinquième année propose, quant à lui ${ }^{4}$, un seul conte algérien (d'origine amazighe), intitulé : Le chêne magique $e^{5}$, coupé en deux parties et pris dans Le grain magique. Contes, poèmes et proverbes berbères de Kabylie, de Marguerite Taos Amrouche.

Le premier projet du manuel de quatrième année, qui comporte trois séquences et qui se propose comme objectif pédagogique (Projet 2), de "lire et d'écrire un conte ${ }^{6}$ se réfère, dans les différentes parties des cours et des activités envisagés, à des contes étrangers. Le manuel de cinquième année propose, en trois séquences, de découvrir le conte : découvrir sa structure narrative, identifier ses particularités et faire parler ses personnages. Il se réfère, lui aussi, à des contes étrangers, et introduit finalement un conte algérien mais toujours dans le même objectif affiché et présenté plus haut.

Une réflexion pourrait alors s'inviter à notre analyse : les manuels et les programmesne sont pas conçus pour proposer à l'élève, ou à l'enfant algérien, des éléments et des paramètres qui font partie de son identité algérienne. Mais nous pouvons comprendre, par contre, qu'il s'agit de manuels et de programmes qui sont pensés dans une approche beaucoup plus interculturelle. Rien, en réalité, ne nous oppose à cette approche qui permet, par ailleurs, énormément de richesses et d'ouverture. Mais il se trouve que nous sommes motivés, dans le présent article, par d'autres ambitions et d'autres motivations.

Il est certainement vrai que :

l'enfant, parce que la vie lui semble souvent déroutante, a le plus grand besoin qu'on lui donne une chance de se comprendre mieux au sein du monde complexe qu'il doit affronter. Il faut donc l'aider à mettre un peu de cohérence dans le 
tumulte de ses sentiments. Il a besoin d'idées qui lui permettent de mettre de l'ordre dans sa maison intérieure et, sur cette base, dans sa vie également.( BETTELHEIM , 1978 : 16).

Il lui faut alors apprendre à vivre en société avec une psychologie équilibrée et consciente. Cet apprentissage, qui commence dès son enfance, serait d'autant plus spontané quand il est donné dans la culture originelle de l'enfant. Car, il s'agit de " la maison » et « du refuge » qui devraient être bien bâtis, bien fondés et bien solides et où les choses et les affaires, qui les meublent, devraient être bien rangées et bien organisées, et ce afin de bien y vivre, de bien s'y sentir et de bien s'y retrouver. Sinon, les sentiments de dépaysement et de non-appartenance pourraient le pousser à la fuite et à la recherche d' "une autre maison» et d' " un autre refuge». Et c'est, alors, le résultat le plus désastreux pour la constitution identitaire de l'enfant.

Initier l'enfant à la vie citoyenne serait une pratique sociale des plus saines, et lui permettre de grandir en s'armant de ses références culturelles et identitaires et notamment à en être conscient serait, selon toute vraisemblance, une obligation sociale des plus urgentes. Le patrimoine culturel algérien est des plus riches en matière de tradition orale: toutes les circonstances de la vie quotidienne sont, à la fois, les occasions et les prétextes pour s'inviter aux temps et aux lieux des personnages des plus étranges et des plus ingénieux.

Ainsi,

pour les hommes, les occasions sont nombreuses : djemaât, réunions de clans ou de quartiers, occasions de travaux agricoles, rencontres dans les cafés, les mosquées, assemblées sur les places publiques à proximité des souks, tous ces lieux sont propices à l'échange verbal qui s'organise autour de légendes, de contes, de proverbes, de paraboles, de devises et d'histoires de familles ou de villages.

Aussi, «les travaux de tissage, les veillées funèbres, offraient aussi des moments intimes» (MEHADJ, 2005) qui permettent la profération des divers récits oraux et qui offrent ainsi des occasions remarquablement enrichissantes et qui consolident les liens familiaux et certainement sociaux.

Il faut, cependant, signaler que cette réalité traditionnelle se trouve sérieusement menacée par l'évolution et le changement de la réalité de la famille algérienne qui a toujours été le berceau des divers genres de la tradition orale. Car, comme nous pourrons le remarquer, les grands-parents -les passeurs et les transmetteurs de cette tradition orale- et notamment dans les grandes villes, ne sont pas aussi présents dans la vie de l'enfant comme c'était le cas autrefois. Leur présence commence à être remplacée par les jardins d'enfants et par d'autres réalités concurrentes (la télévision, l'internet et les autres outils technologiques).

Toutefois, et en dépit de cette présence menacée, la société algérienne, comme toutes les sociétés africaines, maintient, même difficilement, son statut de société où la tradition orale est une tradition multiséculaire et où le fleuve de la bonne parole est très loin d'être asséché. En fait, "aujourd'hui, la narration orale tend à disparaître, des grandes villes du moins, ce qui n'est pas le cas dans les villages algériens où la tradition orale reste tout de même ancrée dans les usages " (Idem). Car il s'agit d'une pratique sociale et culturelle qui garde toujours son importance, même si la société algérienne a pu rencontrer dans son histoire et dans son évolution plusieurs changements qui l'ont touché dans ses différents repères.

14 La tradition orale algérienne est une tradition extrêmement riche en nuances, elle propose des univers imaginaires étonnamment variés et pleins de personnages qui 
reprennent les vices et les qualités des hommes. Elle est également un véritable réservoir des différents genres oraux et notamment le conte. Ce dernier pourrait, lui, par son essence, son organisation et sa construction, véhiculer tous les principes d'une vie saine, positive et constructive : «il(s) célèbre(nt) invariablement les bonnes valeurs: le sérieux, le travail, la générosité, le respect et la tolérance. " (Idem). Autant de leçons et d'enseignements que la tradition orale recèle et dont a besoin la société pour sa stabilité, son développement et son épanouissement.

A notre humble appréciation, un programme éducatif au cycle primaire qui ferait directement et clairement référence à la société algérienne et à sa culture, aurait beaucoup plus d'impact et sur les motivations des élèves dans l'apprentissage du français langue étrangère et sur la constitution et le renforcement de leur dimension identitaire et de leur sentiment d'appartenance. Ce que nous voulons dire par: directement et clairement, c'est une mise en place de programmes qui seraient bâtis sur la présence de contes essentiellement algériens faisant partie des deux langues arabe et amazighe, traduits certainement en français, et ce afin de proposer à l'élève un univers qui correspond à son environnement socioculturel.

La démarche de L.L. Senghor et d'A. Sadji dans La belle histoire de Leuk-Le-Lièvre. Cours élémentaires des écoles d'Afrique Noire (Librairie Hachette, 1953), nous semble des plus pertinentes, des plus cohérentes et des plus conscientes. Car elle propose à l'enfant noir d'apprendre la langue française à travers une démarche pédagogique remarquablement efficace. Le " manuel se compose de récits déjà entendus par l'enfant dans sa langue maternelle et déjà vécus de lui » (SENGHOR et SADJI, 1953 : 4). Il est alors plus facile pour l'élève de s'identifier aux récits et de se représenter les tribulations des actants; en réalité, il part de ce qu'il connait pour aller vers ce qu'il ne connait pas. Il se trouverait, dans cette situation, dans une attitude motivée et confiante et assez confortable pour pouvoir recevoir ce qu'il va lui être transmis.

L'école algérienne aurait-elle besoin d'une telle initiative? Nous le penserions volontiers. Et ses résultats seraient concrètement et sensiblement productifs. Notre petite expérience dans l'enseignement primaire, dans une période de changements et de tâtonnements (entre 2005 et 2008), nous a permis de comprendre à quel point la problématique de l'enseignement, notamment de l'enseignement du français était profonde en Algérie. Au lieu de proposer à l'élève un conte étranger pour la lecture ou pour la connaissance de l'organisation du genre littéraire, et qui ferait partie d'une autre culture ou qui renverrait à une autre réalité sociale que les siennes, il pourrait être plus évident pour lui de passer par des chemins qu'il connait déjà et où il ne serait ni dépaysé ni trop intrigué. Lui proposer donc, des contes algériens (des deux langues arabe et amazighe) qu'il aurait peut être entendus, et qui appartiendraient à ses références socioculturelles, lui permettrait d'abord de mieux suivre l'apprentissage qui lui est proposé, mais notamment de se constituer une identité, certainement composite (MAALOUF, 1998 : 30) mais extrêmement riche en nuances et en ancrages.

Cette démarche serait certainement des plus réalisables et des plus productives, notamment dans un environnement socioculturel où,

«le génie populaire regorge de ce type de narration que les grand-mères transmettaient jadis patiemment autour de l'âtre » et où le «riche trésor oral, véritable socle identitaire, dépeint parfaitement la personnalité collective» (BOUHADIBA, 1994 : 24).

Car il est vrai que les sociétés maghrébines restent des sociétés de fortes traditions orales, et ce même avec l'insertion des nouvelles technologies dans la vie de tous les 
jours et ce que ces nouveautés peuvent introduire et installer comme nouvelles données sociales et culturelles. La société algérienne n'échappe certainement pas à cette réalité socioculturelle.

La tradition orale algérienne regorge également de ces richesses narratives qui sont aussi variées que le sont les régions et les langues ${ }^{7}$ parlées en Algérie. Plusieurs travaux scientifiques $^{8}$ ont pris pour objet d'étude ces richesses et ont pris soin de les collecter et de leur permettre d'accéder à la sphère de l'écrit et même de l'écrit littéraire.

Dans un autre ordre, nous pouvons dire que le trésor oral algérien décrit avec beaucoup de vie la personnalité collective et les aspirations de la société algérienne. Il pourrait très bien véhiculer, à travers les diverses formes orales, les enseignements et les sagesses qui contribueront à former et à façonner le citoyen de demain. Les richesses de la forme "contique", des deux langues arabe et amazighe, ne manqueront certainement pas d'alimenter, enrichir et approfondir l'imaginaire de l'enfant. Ils lui proposeront des récits pleins de châteaux et de palais somptueux, où rois, reines et princesses, tantôt adorables, tantôt méchants et sans pitié, font avancer des univers et chanter des vies. Où le paysan, honnête et courageux, peut avoir la main de la plus belle des princesses et où le riche commerçant, vilain et malhonnête, ne peut même pas rêver de la plus laides des villageoises. Aventuriers futés et grands chasseurs adroits et sages peuplent des villages et des montagnes et alimentent des histoires riches en enseignements et en morales.

Des récits qui traitent de tout ce qui peut animer et motiver l'être humain comme émotions et comme états. Ce sont alors des histoires qui parlent de bonté et de méchanceté, de courage et de lâcheté, de générosité et d'avarice, de gaieté et de tristesse, d'amour et de sacrifice.

Des histoires où les animaux, reproduisant les qualités et les vices des humains, font passer à travers leurs dialogues ainsi que leurs actions des morales porteuses de valeurs et de principes dont la finalité est de proposer les meilleures attitudes à suivre. Ainsi» le chacal et le hérisson, le lièvre et la tortue, le renard et le serpent, la fourmi et la cigale, le roi lion et les autres sujets de la forêt " (KHEIR et al.,2013: 54) transmettent aux hommes les bonnes manières, les éloignent des mauvaises décisions et les orientent vers l'idéal à suivre.

Ces contes sont également porteurs de références intarissables sur les plans moralisateur et éducatif. Une réalité qui pourrait notamment accompagner l'enfant dans son ancrage, dans son évolution et dans son développement identitaire. Car il est question de contes qui « célèbrent invariablement les bonnes valeurs : le sérieux, le travail, la générosité, le respect et la tolérance. " (Idem). Ce qui a pour effet de permettre à l'enfant de faire son initiation dans le monde des responsabilités à travers des récits et des mises en scènes dans lesquels il est complice. Une complicité si chère à l'enfant que tout devient pour lui la continuité de son propre univers imaginaire.

19 Le conte algérien des deux langues arabe et amazighe au cycle primaire ne devrait pas être seulement un outil pédagogique qui permettrait d'accéder à la forme, à la construction narrative et à la portée moralisatrice d'un genre littéraire. La symbolique $\mathrm{du}$ conte et son pouvoir, désormais incontestables, font que son discours, remarquablement universel et étonnamment convaincant,» peut se révéler comme un authentique moyen de pouvoir et d'action aux mains de celles qui le manient comme instrument et comme verbe " (MEHADJI, 2005), s'il est pris et exploité dans ses dimensions politique et idéologique. Et le conte algérien n'échappe pas à cette réalité. Le considérer comme 
un agent idéologique et notamment identitaire dont le pouvoir serait orienté vers la participation àla constitution d'une identité algérienne, aux multiples dimensions et aux diverses richesses, serait alors un choix à prendre et à installer dans le système éducatif algérien.

Le lecteur des contes algériens arabes et amazighes peut retrouver la mise en scène des diverses réalités quotidiennes comme les différences entre les couches sociales, les inégalités et les rivalités qui peuvent exister entre l'une et l'autre. Il y retrouve également les valeurs et les comportements représentatifs des membres de chaque hiérarchie sociale, les différences entre les sexes et ce que ces différences peuvent avoir comme résultats dans le partage des sphères de la vie. La valeur du travail et de l'économie, du savoir, des compétences et des responsabilités y sont également narrées et exposées. Il y est question aussi, des thématiques qui renvoient à la religion, aux libertés, au dialogue et à la cohabitation dans le respect des différences et ce dans l'objectif de construire un individu conscient et responsable.

Ces thématiques qui alimentent le conte algérien peuvent prendre sur un plan politique le rôle de ce qui peut protéger la société et l'immuniser contre ce qui peut menacer sa stabilité et son équilibre identitaires. Le conte permet à la communauté de vivre dans une forme de projection qui prévient, à travers la narration et la mise en scène d'une communauté équivalente, les diverses formes de dérives ou de perturbation. Il serait alors question d'une projection qui programme une :

identité virtuelle qui, dans l'expérience de la parole, s'établit un instant entre le récitant, le héros et l'auditeur engendre selon la logique du rêve une fantasmagorie libératrice. [...] Le conte offre à la communauté un terrain d'expérimentation où, par la voix du conteur, elle s'essaie à tous les affrontements imaginables (ZUMTHOR, 1983 : 53).

Cela est également vrai dans le cas des contes écrits. Le narrateur, qui permet au lecteur d'accéder au monde relaté, offre, à travers les mots, leurs agencements et la construction narrative, une reconstitution d'une société virtuelle équivalente à la sienne et qui pourrait s'ouvrir à toutes les aventures et à toutes les expériences possibles. Cette société virtuelle, équivalente et parallèle, jouerait comme une mécanique qui absorberait et allègerait les problèmes et les paradoxes de la société.

Il faudrait alors une réflexion et un travail qui puissent retrouver les liens permettant de remettre le conte algérien (des deux langues) à la place qui lui revient et de logique et de droit dans les manuels de l'école primaire. Une réflexion et un travail qui auraient pour philosophie la redécouverte de l'histoire et de l'identité algérienne composites et tellement riches et où se conjuguent les mutations et les évolutions de la société.

Le conte algérien des deux langues arabe et amazighe n'a pas cessé d'animer les travaux et les recherches des spécialistes et des universitaires. Mais, il semblerait que ses apports sur les plans idéologique et identitaire ne bénéficient pas de l'intérêt qu'ils méritent. Nous croyons, à la lumière de ce qui a été évoqué dans le présent article, qu'un programme pédagogique qui se propose l'enseignement du français langue étrangère à l'école primaire, et qui serait constitué de contes essentiellement algériens permettrait d'abord une approche plus facile du français. Les élèves de l'école primaire qui reçoivent dès la troisième année l'enseignement du français sont en réalité âgés entre huit et dix ans. Ces enfants auront alors l'opportunité et l'occasion d'accéder à une langue qui a, certes, un statut bien particulier en Algérie et ce, compte tenu d'une certaine réalité historique "butinée ». Mais il reste que le français est loin d'être la 
langue maternelle des algériens, même s'il jouit d'une présence particulière au sein même des familles et dans la vie de tous les jours.

Cet accès au français, fait à travers des chemins et des voies connus par l'enfant algérien, permettrait ensuite de fournir des repères identitaires et idéologiques conformes à son vécu et à son univers culturel. Il n'est nullement question pour nous, dans cet article, de proposer un isolement de l'enfant algérien de ce que les autres univers culturels et civilisationnels pourraient lui offrir ou lui apporter comme différences ou comme enrichissements. Mais il se trouve que notre réflexion gravite autour d'un projet identitaire qui exploiterait les ressources et les potentialités présentes dans le conte algérien des deux langues arabe et amazighe. Car, il s'agit de ce que cette réalité socioculturelle, issue des traditions orales algériennes ${ }^{9}$, pourrait exercer comme impact et comme influence sur la constitution psychologique et notamment identitaire de l'enfant algérien futur citoyen et acteur dans sa société.

\section{BIBLIOGRAPHIE}

BETTELHEIM, B. 1978, Psychanalyse des contes de fées, Paris : Editions Robert Laffont.

BOUHADIBA, A. 1994, L’imaginaire maghrébin, Tunisie : Cérès.

GRANDGUILLAUME, G. 2001, Les débats et les enjeux linguistiques, in MAHIOU A., HENRY J.-R. (dir.), Où va l'Algérie ? France, KARTHALA-IREMAM, p. 273-287

KHEIR A., TIFOUR T. et AIT AMAR M. O. 2013, L'interculturel et l'enseignement/apprentissage du texte littéraire : le cas du conte, Synergies Chine $\mathrm{n}^{\circ} 8$.

MAALOUF, A. 1998, Les identités meurtrières, Editions Grasset \&Fasquelle.

MEHADJI Rahmouna' Le conte populaire dans ses pratiques en Algérie, L'Année du Maghreb [Online], II | 2005-2006, Online since 08 July 2010, connection on 16 January 2016. URL : http://

anneemaghreb.revues.org/151; DOI : 10.4000/anneemaghreb.151

MUKENE, P. 1988, L'ouverture entre l'école et le milieu en Afrique noire. Pour une gestion pertinente des connaissances, Suisse, Editions universitaires de Fribourg.

SENGHOR Léopard S., SADJI A. 1953, La belle histoire de Leuk-Le-Lièvre. Cours élémentaires des écoles d'Afrique Noire, Librairie Hachette.

ZUMTHOR, P. 1983, Introduction à la poésie orale, Paris, Seuil.

Français, $3^{\text {ème }}$ année primaire. Manuel officiel, première édition 2017-2018. MEDJAHED Leila, Maitre de conférences, FERHAT Mouloud, Inspecteur de l'enseignement primaire, GHERBAOUI Mohamed, Inspecteur de l'enseignement primaire, KOUADRI Mohamed, Professeur de l'enseignement primaire.

Mon premier livre de français, $3^{\text {ème }}$ année primaire. Manuel officiel, 2013-2014. M'HMSADJI-TOUNSI Mina, I.E.E.F, BEZAOUCHA Anissa, P.E.S, MAZOUZI GUESMI Sadjia, M.E.F.

Mon livre de français, $4^{\mathrm{e}}$ Année Primaire, Manuel officiel, 2014-2015. Mmes. KORICHE Hamida

(I.E.E.F), DADDA Aïcha (Universitaire) et M. IMMAMOUINE M'Hmed. 
Mon livre de français, $5^{\mathrm{e}}$ Année Primaire. Manuel officiel, 2013-2014. SRITI Lamine (inspecteur de l'éducation nationale), Ferrah Sacia (MEF), ADJROUD (PEF), NOUI Fouzia (MEF), NADJI Mohamed (MEF). Revue et corrigé par : BELHADJ MILOUD Inspectrice de l'enseignement primaire), BOUHI Aoumria (Inspectrice de l'enseignement primaire).

\section{NOTES}

1. ${ }^{1}$ Nous désignons par cette expression les contes issus de la tradition orale arabe et ceux issus de la tradition orale amazighe, et nous voulons dire par les contes de la langue amazighe, ceux issus des traditions orales : chaoui, kabyle, mozabite, chenoui et touareg.

2. ${ }^{2}$ Mais il y a un texte (p. 62, du manuel de troisième année) adapté d'après BENGHEDIH KHATI Rabéa et intitulé Le petit nuage curieux. C'est un texte proposé sans précision du genre.

3. ${ }^{3}$ Voir à ce sujet: Mmes. KORICHE Hamida (I.E.E.F), DADDA Aïcha (Universitaire) et M. IMMAMOUINE M'Hmed, Mon livre de français, $4^{\mathrm{e}}$ Année Primaire, Manuel officiel, 2014-2015, p. 14 puis p. 94.

4. ${ }^{4}$ Mon livre de français, $4^{\mathrm{e}}$ Année Primaire, Manuel officiel, 2014-2015. Mmes. KORICHE Hamida (I.E.E.F), DADDA Aïcha (Universitaire) et M. IMMAMOUINE M'Hmed.

5. ${ }^{5}$ Voir à ce sujet: SRITI Lamine (inspecteur de l'éducation nationale), Ferrah Sacia (MEF), ADJROUD (PEF), NOUI Fouzia (MEF), NADJI Mohamed (MEF). Revue et corrigé par : BELHADJ MILOUD Inspectrice de l'enseignement primaire), BOUHI Aoumria (Inspectrice de l'enseignement primaire), Mon livre de français, $5^{\mathrm{e}}$ Année Primaire. Manuel officiel,2013-2014. p. 55, puis 65.

6. ${ }^{6}$ Voir à ce sujet: Mmes. KORICHE Hamida (I.E.E.F), DADDA Aïcha (Universitaire) et M. IMMAMOUINE M'Hmed, Mon livre de français, $4^{\mathrm{e}}$ Année Primaire, Manuel officiel, 2014-2015.

7. ${ }^{7}$ Nous prenons le mot langue ici dans sa conception première, celle de moyen de communication partagé par les membres d'une même communauté et nous pensons à l'arabe et au tamazight.

8. ${ }^{8}$ Nous citons par exemple les travaux de l'anthropologue et ethnologue allemand Leo Frobenius (1873-1938) sur les contes kabyles entre 1913 et 1914. Profitant des missions scientifiques en Afrique, notamment en Algérie, il a pu collecter et traduire dans quatre volumes des contes kabyles. Ces ouvrages, parus en langue allemande entre 1922 et 1923, ont pu être publiés avec la traduction en français d'Ariette Roth puis celle de Mokran Fetta : Contes kabyles. Tome 1 : Sagesse (Broché, 1995), Contes kabyles, Tome II : Le Monstrueux (Broché, 2000),Contes kabyles. Tome III : Le Fabuleux (Broché, 1997) et enfin Contes kabyles. Tome IV : Autres contes fabuleux, (Broché, 1998).

Nous retrouvons aussi Le grain magique. Contes, poèmes et proverbes berbères de Kabylie (1966, Poche, 2007) de Marguerite Taos Amrouche (1913-1976), Contes algériens de Christiane Chaulet Achour et Zineb Ali Benali (Ed. L'Harmattan, 1990) ou encore Le Figuier magique et autres contes algériens de Michele Galley, livre-CD avec la voix de Aouda (Ed. Geuthner, 2003).

Nous pouvons citer également des travaux universitaires importants dans la sauvegarde du patrimoine oral algérien comme par exemple le travail de Rahmouna Mehadji, maître de conférence à l'Université d'Oran La Sénia et chargée de recherche sur le patrimoine immatériel algérien au CRASC (Centre de recherche en anthropologie sociale et culturelle). Elle est aussi l'auteur d'une participation sur Le conte populaire dans ses pratiques en Algérie (2007), où elle a répertorié et analysé les diverses formes et les différents lieux de profération du conte et ses acteurs. Ce projet s'est référé au travail de collecte des contes qu'elle a entrepris dans la région d'Oran entre 1998 et 2005.

Il est également intéressant de mentionner le travail remarquablement conduit de Goual Doghmane Fatima : Etude sémio-narrative des contes Touareg production féminine. Un travail qui se distingue par sa grande pertinence et qui s'est concentré sur des contes touaregs, une réalité 
culturelle constituante de l'identité algérienne. Ces travaux et ces réflexions, et tant d'autres, contribuent, de manière très significative, à maintenir en vie un patrimoine immatériel hautement sensible, dans un environnement social et culturel gouverné par d'autres circonstances que celles qui ont pu lui donner le jour.

9. ${ }^{9}$ Nous sommes tentés, à l'issu de cet article, de parler de «traditions orales algériennes » au pluriel. Car, il s'agit d'univers d'expressions et d'imaginations variées et qui peuvent se référer à des traditions et à des coutumes qui le sont également.

\section{RÉSUMÉS}

Le conte est une réalité sociale, culturelle et civilisationnelle remarquablement protectrice et enrichissante. Issu des traditions orales, il a pu s'offrir une position et un statut bien particuliers dans l'écrit et notamment dans l'écrit littéraire. Lui donner une place et un rôle dans les manuels et les programmes pédagogiques est une entreprise qui s'est montrée pleine de potentialités. Le conte algérien des deux langues arabe et amazighe, est un outil pédagogique qui devrait retrouver sa place au sein du système éducatif, non seulement en tant que genre littéraire, mais également en tant qu'agent identitaire qui aurait pour objectif de participer à la constitution d'une identité bien ancrée et consciente.

The tale is a social reality, cultural, educational and civilized, remarkably protective and rich. It came from the oral traditions and it gained a special position and status in writing especially in the literary ones. It save it an important place and role in the manuals and the pedagogical programmers that undertakings whichextremely displayed full of potentials. The Algerian talein Arabic and Tamazight, is a pedagogical tool that has to gain its place within the educational system. Not only as a literary sort but also as an ideological agent which has the objective to participate in the establishing of family rooted and conscious identity.

\section{INDEX}

Keywords : tale, pedagogy, identity, culture, civilization

Mots-clés : conte, identité, pédagogie, culture, civilisation

\section{AUTEUR}

\section{ATTEF BOUZIDI}

Université d'Annaba 